\title{
Research on Assessing Public Web-chat Accounts based on the Dispersed Gray Model and Comprehensive Evaluation Model
}

\author{
Yifeng Dou, a, Wang Zhang ${ }^{2, b}$, Qin Hu ${ }^{3, ~ c}$, Hailing Xiong ${ }^{4, d}$ \\ ${ }^{1}$ College of Computer and Information Science, Southwest University, Chongqing, 400715, China \\ ${ }^{2}$ College of Plant Protection, Southwest University, Chongqing, 400715, China \\ ${ }^{3}$ College of Plant Protection, Southwest University, Chongqing, 400715, China \\ ${ }^{4}$ College of Computer and Information Science, Southwest University, Chongqing, 400715, China \\ aemail: 1204840733@qq.com, bemail:261729148@qq.com, cemail:976227524@qq.com \\ demail:xionghl@swu.edu.cn
}

\begin{abstract}
Keywords: DGM(2,1); Structure Equation; Comprehensive Evaluation Method; Diffusion Theory of Innovation
\end{abstract}

\begin{abstract}
The mathematical models are built to predict the growth trend of public accounts corresponding to different fields, and to evaluate whether Web-Chat operate their platforms effectively. Four domains of public account information are chose which are representative in public web-chat accounts, these areas are education, government, media and network. Secondly, the number information of those fields are used and handled them. Finally, based on the dispersed gray model as the foundation, that is $\operatorname{DGM}(2,1)$. What's more, error rationality $\mathrm{C}$ is calculated, that are $0.43,0.42,0.53,0.61$ and the error probability $\mathrm{P}$ are $0.89,0.86,0.75,0.88$ respectively in four different fields. We found that the model $\operatorname{DGM}(2,1)$ is better than $\operatorname{GM}(1,1)$ in forecasting rising trend of Web-Chat in different fields. In order to evaluate whether Web-Chat users operate their platforms effectively, the model is set up which combines the structure equation with comprehensive evaluation method to solve the problem. To test the model, five different fields are pick up, namely, government, education, media, website and celebrity. The result of the model shows that users in the fields of education and celebrity run their public web-chat accounts exceedingly effectively and the public accounts don't work well in other three fields.
\end{abstract}

\section{Introduction}

Web-chat is a mobile text and voice message communication service developed by Tencent in China, first released in January 2011. Up to August 2014, there have been 438 million active domestic users and 70 million foreign loggers. It is worth noting that the users of public web-chat account allowed to send one message to common people everyday, but only a very few public accounts use the only chance to sent their group messages to common people. the majority of users of public account would send valuable news which they think worthy of attention and interesting messages which they think that audience may be interested in ,based on their own topics and target content. It is important for the companies to analyze whether they operate their platforms effectively and whether they would have made better use of the public platforms since there are numerous public accounts in various fields.

In this paper we have researched to build mathematical models to solve the following tasks: (1) Looking for references to analyze the fields of Web-chat public accounts and model to predict the growth trend of public accounts corresponding to different fields. (2) Setting up a model to analyze whether public accounts in different fields are effectively run and explain the advantages and disadvantages of model.

\section{The establishment and solution of model in task 1}

In order to predict the growth trend of number of media, government, education, network public 
account registrations, we choose 8 consecutive months of public accounts in each field on 2015 September as the original sequence, and constructed the forecasting model DGM $(2,1)[1]$.

1. Each field of the original sequence $X_{k}^{(0)}$ is as follows:

Tab.1 The registered amount of media, government, education, network

\begin{tabular}{|c|c|c|c|c|c|c|c|c|}
\hline & 2015.0 & 2015.0 & 2015.0 & 2015.0 & 2015.0 & 2015.0 & 2015.0 & 2015.0 \\
& 9.04 & 9.05 & 9.06 & 9.07 & 9.08 & 9.09 & 9.10 & 9.11 \\
\hline Media & 209 & 285 & 364 & 653 & 799 & 1107 & 2579 & 3107 \\
\hline Government & 227 & 116 & 142 & 318 & 364 & 525 & 727 & 1013 \\
\hline Education & 215 & 256 & 264 & 241 & 422 & 486 & 502 & 574 \\
\hline Website & 221 & 416 & 685 & 1022 & 955 & 1337 & 2254 & 2130 \\
\hline
\end{tabular}

To weaken the fluctuation and randomness of sequences, we accumulated the original sequence and got the new data sequence $X_{k}^{(1)}$ :

$$
X_{k}^{(1)}=\left\{x_{k}^{(1)}(1), x_{k}^{(1)}(2), \ldots, x_{k}^{(1)}(n)\right\}, k=1,2,3,4
$$

2. Then the differential equation DGM(1.1)is obtained:

$$
\frac{d^{2} x_{k}^{(1)}}{d t^{2}}+a_{k} \frac{d x_{k}^{(1)}}{d t}=b_{k}, k=1,2,3,4
$$

Here $\mathrm{a}$ and $\mathrm{b}$ are both determined parameters.

3. Discrete the formula(10),we got:

$$
B_{k}=\left[\begin{array}{cc}
-x^{(0)}(2) & 1 \\
-x^{(0)}(3) & 1 \\
\ldots & \ldots \\
-x^{(0)}(n) & 1
\end{array}\right] Y_{k}=\left[\begin{array}{c}
\alpha^{(1)} x_{k}^{(0)}(2) \\
\alpha^{(1)} x_{k}^{(0)}(3) \\
\ldots \\
\alpha^{(1)} x_{k}^{(0)}(n)
\end{array}\right]=\left[\begin{array}{c}
x_{k}^{(0)}(2)-x_{k}^{(0)}(1) \\
x_{k}^{(0)}(3)-x_{k}^{(0)}(2) \\
\ldots \\
x_{k}^{(0)}(n)-x_{k}^{(0)}(n-1)
\end{array}\right] \mathrm{k}=1,2,3,4
$$

4. We calculated gray parameters $a_{k}$ a by the least square method:

$$
a_{k}=[a, b]^{T}=\left(B^{T} B\right)^{-1} B^{T} Y, k=1,2,3,4
$$

Tab.2 Gray parameters

\begin{tabular}{|c|c|c|c|c|}
\hline $\begin{array}{c}\text { Fields/paramet } \\
\text { ers }\end{array}$ & Media & Government & Education & Website \\
\hline$a_{k}$ & -0.3265 & -0.3567 & -0.2054 & -0.1562 \\
\hline$b_{k}$ & -0.8384 & -51.0186 & -29.2440 & 76.3842 \\
\hline
\end{tabular}

5. Taking $a_{k}, b_{k}$ into (10), we got

$$
x_{k}^{(1)}(t+1)=\left(\frac{b_{k}}{a_{k}^{2}}-\frac{x_{k}^{(0)}(1)}{a_{k}}\right) e^{-a_{k} t}+\frac{b_{k}}{a_{k}}(t+1)+\left(x_{k}^{(0)}(1)-\frac{b_{k}}{a_{k}}\right) \frac{1+a_{k}}{a_{k}} \mathrm{k}=1,2,3,4
$$

6. Distance $x_{k}^{(1)}(t+1)$ and $x_{k}^{(1)}(t)$, we got $x_{k}^{(0)}(t+1)$ as follows:

$$
x_{k}^{(0)}(t+1)=\alpha_{k}^{(1)} x_{k}^{(1)}(t+1)=x_{k}^{(1)}(t+1)-x_{k}^{(1)}(t) \mathrm{k}=1,2,3,4
$$


Tab.3 Approximate registrations in government, education, media network

\begin{tabular}{|c|c|c|c|c|c|c|c|c|}
\hline \multirow{2}{*}{ Times/Fields } & 2015.0 & 2015.0 & 2015.0 & 2015.0 & 2015.0 & 2015.0 & 2015.0 & 2015.0 \\
& 9.04 & 9.05 & 9.06 & 9.07 & 9.08 & 9.09 & 9.10 & 9.11 \\
\hline Media & 207 & 244 & 339 & 469 & 650 & 901 & 1249 & 1732 \\
\hline Government & 85 & 101 & 144 & 206 & 294 & 421 & 601 & 858 \\
\hline Education & 73 & 81 & 99 & 122 & 149 & 183 & 225 & 276 \\
\hline Website & 710 & 769 & 899 & 1050 & 1228 & 1436 & 1678 & 1962 \\
\hline
\end{tabular}

7. To test the gray model, the steps are as follows:

(1) Calculating $e_{k}^{(0)}(t)$ between $x_{k}^{(0)}$ and $x_{k}^{(0)}(t)$ :

$E_{k}=\left[e_{k}^{(0)}(1), e_{k}^{(0)}(2), \ldots, e_{k}^{(0)}(n)\right]=x_{k}^{(0)}-x_{k}^{(0)}$

$e_{k}^{(0)}(t)=x^{(0)}(t)-x^{\prime(0)}(t), t=1,2, \ldots, n ; k=1,2,3,4$.

(2) Calculating the mean and variance $S_{1, k}^{2}$ of the original series $x_{k}^{\prime(0)}$ :

$$
\begin{aligned}
& S_{1, k}^{2}=\frac{1}{n} \sum_{t=1}^{n}\left[x_{k}^{(0)}(t)-\overline{x_{k}}\right]^{2} ; S_{2, k}^{2}=\frac{1}{n} \sum_{t=1}^{n}\left[e_{k}(t)-\overline{e_{k}}\right]^{2} \\
& -\overline{x_{k}}=\frac{1}{n} \sum_{t=1}^{n} x_{k}^{(0)}(t), e_{k}=\frac{1}{n} \sum_{t=1}^{n} e_{k}(t) ; k=1,2,3,4 .
\end{aligned}
$$

(3) Calculate variance ratio:

$$
C_{k}=\frac{S_{2, k}}{S_{1, k}} ; k=1,2,3,4 \text {. }
$$

(4) Calculate small error probability:

$$
p_{k}=P\left\{\left|e_{k}(t)-\overline{e_{k}}\right|<0.6745 S_{1, k}\right\}
$$

Tab.4 Variance ratio $C_{k}$ and small error probability $p_{k}$

\begin{tabular}{|c|c|c|c|c|}
\hline Fields/Parameters & Media & Government & Education & Website \\
\hline$C_{k}$ & 0.5304 & 0.1868 & 0.5349 & 0.4504 \\
\hline$p_{k}$ & 0.7500 & 1.0000 & 0.8750 & 0.8750 \\
\hline
\end{tabular}

(5) Grey model precision test table as follows:

Tab.5 Precision inspection class reference list

\begin{tabular}{|c|c|c|}
\hline Model accuracy grade & Variance ratio C & Small error probability $\mathrm{P}$ \\
\hline One-level (good) & $\mathrm{C}<=0.35$ & $0.95<=\mathrm{P}$ \\
\hline Two-level (qualified) & $0.35<\mathrm{C}<=0.5$ & $0.80<=\mathrm{P}<0.95$ \\
\hline Three-level (general) & $0.5<\mathrm{C}<=0.65$ & $0.70<=\mathrm{P}<0.80$ \\
\hline Four-level (unqualified) & $0.65<\mathrm{C}$ & $\mathrm{P}<0.70$ \\
\hline
\end{tabular}

(6) Comparing with the precision test table, we got the prediction effect in media, government, education, network as follows:

Tab.6 The prediction effect in media, government, education, network

\begin{tabular}{|c|c|c|c|c|}
\hline Parameters & Media & Government & Education & Website \\
\hline$k$ th class & third & first & third & second \\
\hline
\end{tabular}

8. Using DGM $(2,1)$ model to predict the Micro message public account in four fields for the next ten days: 
Tab.7 The prediction of Micro message public account in four fields for the next ten days

\begin{tabular}{|c|c|c|c|c|c|c|c|c|c|c|}
\hline & $\begin{array}{c}2015 . \\
09.12\end{array}$ & $\begin{array}{c}2015.0 \\
9.13\end{array}$ & $\begin{array}{c}2015 . \\
09.14\end{array}$ & $\begin{array}{c}2015 . \\
09.15\end{array}$ & $\begin{array}{c}2015 . \\
09.16\end{array}$ & $\begin{array}{c}2015 . \\
09.17\end{array}$ & $\begin{array}{c}2015 . \\
09.18\end{array}$ & $\begin{array}{c}2015 . \\
09.19\end{array}$ & $\begin{array}{c}2015 . \\
09.20\end{array}$ & $\begin{array}{c}2015.0 \\
9.21\end{array}$ \\
\hline Media & 2400 & 3327 & 4611 & 6391 & 8859 & 12279 & 17020 & 23591 & 32700 & 45326 \\
\hline Government & 1225 & 1750 & 2500 & 3527 & 5102 & 7289 & 10412 & 14874 & 21248 & 30355 \\
\hline Education & 340 & 417 & 512 & 629 & 772 & 948 & 1164 & 1429 & 1754 & 2154 \\
\hline Website & 2294 & 2681 & 3134 & 3664 & 4284 & 5008 & 5854 & 6844 & 8001 & 9353 \\
\hline
\end{tabular}

9. The overall trend graph in government, education, media ,network registration are shown in Fig.1, respectively:

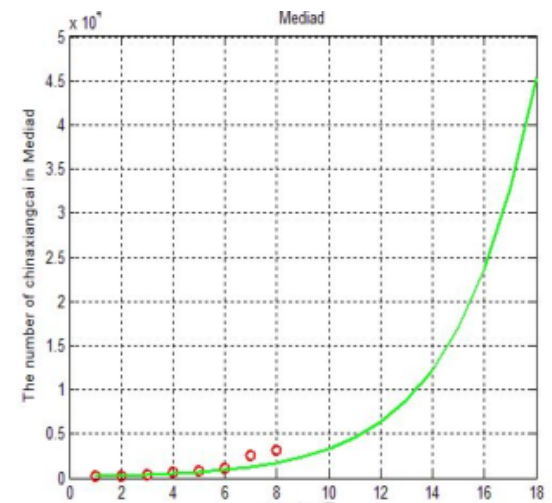

(a)Media

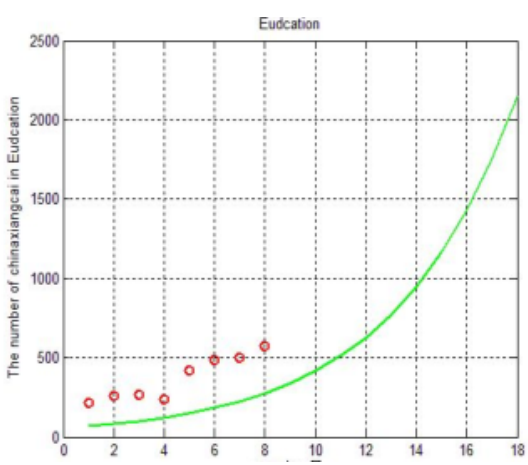

(c)Education

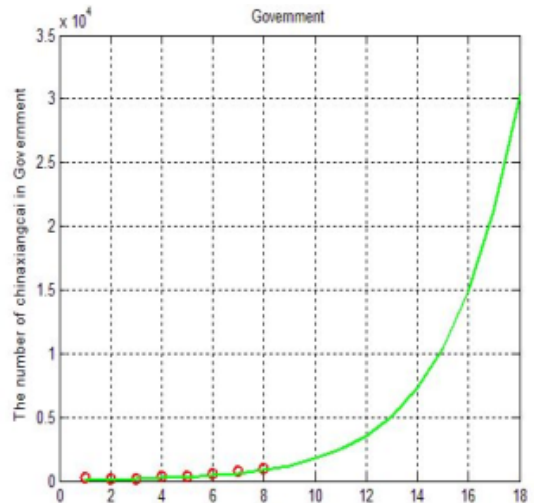

(b)Government

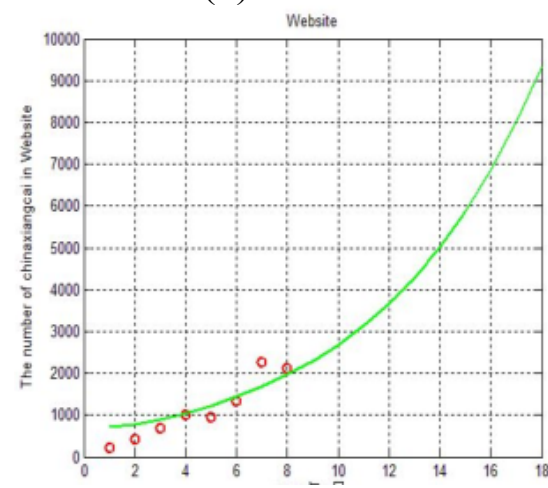

(d)Website

Fig. 1 The overall trend graph in four fields

\section{The establishment and solution of model in task 2}

Taking the six factors expressed by "True" and "False" in the data table to regard as the users' running strategies. And coverting the "True" and "False" into the binary value "1" and " 0 " respectively. It is showed in the Tab.8.

Tab.8 The way of converting managerial tactics into binary value

\begin{tabular}{|c|c|}
\hline Whether the temporary logger watch out the user & $X_{1}$ \\
\hline Whether someone is allowed to send me private messages & $X_{2}$ \\
\hline Whether there is some geological information on the account & $X_{3}$ \\
\hline Whether the account is authenticated by web-chat & $X_{4}$ \\
\hline Whether all people are allowed to comment on my web-chat & $X_{5}$ \\
\hline Whether the user watch out the temporary logger & $X_{6}$ \\
\hline
\end{tabular}

Tab.9 The result of comparative

\begin{tabular}{|c|c|c|c|c|c|c|}
\hline Running strategies & $X_{1}$ & $X_{2}$ & $X_{3}$ & $X_{4}$ & $X_{5}$ & $X_{6}$ \\
\hline State & False & False & True & True & True & False \\
\hline Binary value & 0 & 0 & 1 & 1 & 1 & 0 \\
\hline
\end{tabular}

Through referring to [2] and discussing, the importance order the six running strategies are listed 
as following: $X_{5}>X_{4}>X_{2}>X_{3}>X_{6}>X_{1}$. So define the running value of the public web-chat account: $\xi=X_{5} X_{4} X_{2} X_{3} X_{6} X_{1}$. The running value of Tab.9 $\xi=(110100)_{2}=(52)_{10}$. The running value would have influence on the number of fans $Y_{1}$, the times of being watched out $Y_{2}$, the times of being collected $Y_{3}$ and the times of being transmitted $Y_{4}$. Regard as latent variable and $Y_{1}, Y_{2}, Y_{3}$ and $Y_{4}$ as manifest variables. Construct the measurable equations [3] are shown in Fig.2:

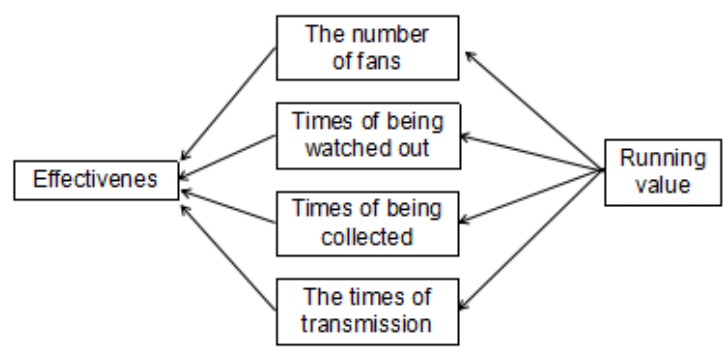

Fig.2 The measurable equations

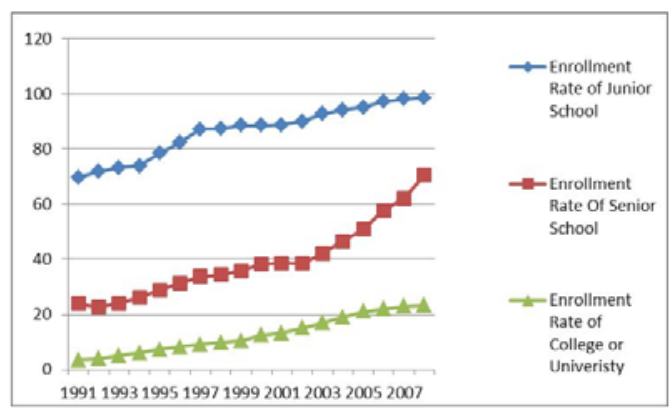

Fig. 3 the enrollment rates of different type school

Adopt the method of "max-min" to eliminate the magnitude differences of data:

$$
Y_{i, j}^{*}=\frac{Y_{i, j}-\min _{j}\left\{Y_{i, j}\right\}}{\max _{j}\left\{Y_{i, j}\right\}-\min _{j}\left\{Y_{i, j}\right\}}
$$

the measurable equation is established as below

$$
Y_{i}=\lambda_{i} \xi+\delta_{i}, i=1,2,3,4 \text {. }
$$

Here $\delta$ is an error, which is not associate with $\mathrm{Y}$ and $\xi, \lambda$ is the correlation coefficient, which means to what degree the latent variable would influence the manifest variables. In this paper, effectiveness was assessed from the the manifest variables and the comprehensive evaluation model was set up to solve the problem. Weight $\omega_{i}$ is determined by $\lambda_{i}$ :

$$
\omega_{i}=\frac{\lambda_{i}}{\sum_{i=1}^{4} \lambda_{i}}
$$

The scores determined by manifest variables:

$$
S=\sum_{i=1}^{4} \omega_{i} \sum_{j=1}^{1000} Y_{i, j}
$$

Effective Managerial Value of Public Web-chat Account:

$$
v=\frac{s}{\sum_{j=1}^{1000} \xi_{j}}
$$

If $v>0.5$, the running strategies of the users in specific field are effective in the public web-chat account [4]. If $v<0.5$, the running strategies of the users in specific field are ineffective in the public web-chat account.

In virtue of MATLAB [5], results of managerial effectiveness of public web-chat accounts were got through coding according to the mathematical model. And there are five fields that are chosen in this paper, respectively, Government, Education, Website, Media, Celebrity.

Tab.10 Five fields

\begin{tabular}{|c|c|c|c|c|c|}
\hline & Media & Government & Education & Website & Celebrity \\
\hline$v$ & 0.1491 & 0.3828 & 0.6366 & 0.1761 & 0.5991 \\
\hline Yes or No & No & No & Yes & No & Yes \\
\hline
\end{tabular}

Tab.10 shows that in the educational field and celebrities'field, the public web-chat accounts'running are pretty successful and effective. Analysis of results are as follows:

(1) Media and website field:Website and media mainly utilize the Internet to attract common people and their strong points are internet, television, newspapers and radio-broadcast, which mean 
they can't effectively run the public accounts. Besides, they did the same as what the government did, not allowing others to comment and having less communications with blog-watchers. The web-chat was originally served to mobile phone, not internet or others. And it can't easily run effectively in these two fields.

(2) Government field: The reasons why the public web-chat accounts didn't run effectively in government field are that government departments create web-chat account just to issue some policies and notices and they don't allow anyone to comment on their words on public accounts or reveal their location information or communicate with common people. If the web-chat accounts are wanted to run effectively in government field, some authorities have to change their managerial strategies. Such as, interacting more with fans and allowing fans to make legal comments on their blogs.

(3) Education field: From the table, we could found that the education field was the most effective to run the public web-chat account. Because in china, more and more people attach importance to educational field and think knowledge will be more and more important. From the Fig.3, it's clearly to be seen that the enrollment rates of junior school, senior school and university are rising year by year. Another reason why the education was the most effective field was that most public web-chat accounts would allow people to comment and reveal their locations and have a registered account. And nowadays, almost every contemporary student has a mobile phone and likes to share their ideas about educational systems by using mobile phone. So the platform of public web-chat accounts runs effectively in education field.

(4) Celebrity field: Celebrity is another effective field. Because in the contemporary society, the star craze is around everywhere. The most users of web-chat public account are young people, however, young people like to pay much attention to celebrities, especially some famous singer stars and movie stars. Meanwhile, most celebrities are willing to communicate with their fans through web-chat account.

The combined method of structure equation and comprehensive evaluation method absorbs the both merits and eliminates respective defects. It can achieve a balance between objectivity and subjectivity and judge effectiveness thoroughly and get a fair resolution to the problem. When choosing the endogenous and indigenous factors, it's easy for judges to make mistakes and be confused with some ambiguous factors. fixing the thresh value totally depends on statistics data, which could neglect the judges'opinions.

\section{Analysis of results}

From the second part, we can find that the among web-chat public channels the channels about education run most successfully. Mathematical Modeling channel is one of them. So we take successful some experiences of these educational web-chat channels to make following plans.

1. Position of mathematical modeling web-chat channel

According to position of effective public education channels, we would like to define our channel to a subscription. Subscription is a kind of counts a type of public platform, which provides information and news for users and can send a message to user every day. The messages will be displayed in the user's subscription document folders.

2. Time and frequency

According to the operations of most operators which are more successful Web-chat public channel in education, we decide to push a message every day.

3. The way of sending

To send information to the users, we choose two forms, which is single and multi-graphic. There are also links to other websites of video sending.

4. Content

(1) Interactive activities

We will occasionally hold small-scale activities to close followers and increase interaction, and have a discussion on the topic of algorithms, models or procedures; and we can also hold some small games such as information retrieval contest. 
(2) League

With free training as well as strong team modulus intelligence group to attract users to join the Society of mathematical modeling, such as the training of various commonly-used software in mathematical modeling, mathematical models and mathematical modeling thesis writing and so on.

(3) Advertisement

Invite others to pay attention to the group through various websites, mathematical modeling or school qq groups with an interesting two-dimensional code. Publish articles or screen link with a two-dimensional code, too.

(4) The introduction of various mathematical modeling contests

Push the time of various mathematical modeling and game time. Before the game, push the countdown to remind participants of getting fully prepared. And basic training of mathematical model before the game is for the participants' convenience.

(5) Something else

Invite the winners of Mathematical modeling competition to do lessons about their experiences. Share good articles and experience with others.

5. Custom Menu

The users will achieve the custom menu after the certificating of Web-chat. Through this menu, such sub-menu like group introduction, public account's establishment, purpose, welfare, activities etc. could be custom by this menu. According to these different categories, the secondary menu could be set under the sub-menu, such as Micro-community, Activities. Company community could be applied by the official website. The users could join in the topic post and discuss through lower function "post", which provides a platform to these topics, increasing the interface and interest such as the discussion of some innovative algorithm.

\section{Conclusion}

Based on the dispersed gray model and structure equation model as the foundation, to predict the growth trend of public accounts corresponding to different fields, and to evaluate whether Web-Chat operate their platforms effectively. Task 1 we calculated error rationality $\mathrm{C}$ and the error probability $\mathrm{P}$ respectively in four different fields, and finding the model we built has good prediction effect. And the combined method of structure equation and comprehensive evaluation method absorbs the both merits and eliminates respective defects for task 2 . It can achieve a balance between objectivity and subjectivity and judge effectiveness thoroughly and get a fair resolution to the problem. However, because the collected data is limited, so the model can only predict the growth trend of public accounts corresponding to different fields in a short time. When choosing the endogenous and indigenous factors, it's easy for judges to make mistakes and be confused with some ambiguous factors. fixing the thresh value totally depends on statistics data, which could neglect the judges'opinions. In the future, we will improve them in the future of the study.

\section{Acknowledgement}

In this paper, the research was sponsored by the 7th "Kehui cup" Competition of Chongqing Postgraduate Student Innovation and Entrepreneurship (Project No. 201571110).

\section{References}

[1] Shao Y, Su H J. On Approximating Grey Model DGM(2,1)[J]. Aasri Procedia, 2012, 1:8-13.

[2] H.R.Kells. The assessment of managerial effectiveness at universities in developing countries[J]. International Institute for Educational Planning, 1991, 9(6):9-18.

[3] Do Nascimento S, Beuren I M. Managerial Effectiveness as Measured by Achievement of Goals Established in the Reward System and its Relation with Psychological Empowerment[J]. Innovar, $2015,25$. 
[4] Gazbar Y. MODELS OF DIFFUSION, ADOPTION, INNOVATION AND ACCEPTANCE OF A NEW TECHNOLOGY, AND SOCIAL COMMUNICATION[J]. Interdisciplinary Journal of Contemporary Research in Business, 2013.

[5] Gilat A. MATLAB : an introduction with applications[M]. J. Wiley \& Sons, 2015. 